\title{
Poster Presentations: A Presenting Opportunity in Conferences for Medical Students
}

\author{
Hulegar A. Abhishekh, ${ }^{1}$ Neeraj Tandon, ${ }^{2}$ Sunil Rangarajan,, ${ }^{3}$ Aisha Gharaibeh, ${ }^{4}$ Francisco J. Bonilla-Escobar. ${ }^{5}$
}

\begin{abstract}
Presenting research findings in conferences develops scientific abilities in early career scientists, facilitates early dissemination of information and provides opportunities for learning different research approaches.' A participant can learn about unpublished research which stimulates ideas much before the article goes through lengthy process of publication. Critical feedback and comments from peers might help in the process of improving a developing manuscript. Conferences provide a platform to build one's network and meet global leaders and new collaborators in one's field. For early career residents and fellows, this can improve the chance of getting long term mentorship that results in overall betterment of career development by various means like getting good opportunities to get into fellowship or early faculty jobs.
\end{abstract}

Unfortunately, medical students and specialists lack the training related with the process of writing an abstract, organizing a poster or preparing for a scientific conference, in general.2,3 The International Journal of Medical Students (IJMS) as it aims to enhance the role of medical students in research publication, also believes in improving the chances of medical students and early physicians to participate in conferences by sharing the tips and information mentioned below. ${ }^{4}$

Conferences vary from small conferences such as symposia, those which discuss specific topics, have narrow research focuses, or are open for advanced research, to large conferences such as society meetings, those which have broad coverage of a field, are open for beginner researchers or interdisciplinary research. Choosing the right conference to participate in is the first step towards a fruitful conference experience.

The next step for the participant is to prepare the abstract. Recently, the STROBE (STrengthening the Reporting of OBservational studies in Epidemiology) initiative published a checklist for conference abstracts which includes a list of items and recommendations for abstracts to gain better positions in research conferences (Available from: http://www.strobe-statement.org/ index.php?id=available-checklists. . ited 2014 Jun 22).

Generally, all conferences provide an opportunity to present as either a poster or oral presentation. Both kinds of presentations have distinct advantages. Poster presentations have the potential to attract larger audiences compared to oral presentations. Additionally, they provide opportunity for longer dis- cussion compared to an oral presentation where time frame is limited to 10-15 minutes.

Another important difference between the two types of presentation is the way in which the presenter engages their audience. Poster presentations receive a diverse set of visitors who may be interested in particular parts of the poster relevant to their field of study or expertise. Since it is more interactive, it becomes challenging to the presenter to quickly gauge the interest and attention span of the audience and modify his presentation of the data. This makes it all the more important for the presenter to understand every bit of data that is presented along with the accurate in-depth methodology. Although oral presentations allow for more diagrammatic representation of results with the inclusion of supplementary data, which usually gets eliminated in poster presentations due to space constraints, there will be very few slots for oral presentations compared to poster presentations and many are given to faculty members. We recommend poster presentation for early career professionals. Details about oral presentation can be found elsewhere.

Presenting poster provides several opportunities. Authors can find the opportunity to develop a national or international reputation, associate their name with a topic, generate network, find a job or a promotion, obtain funding and find help with manuscript preparation. ${ }^{6}$

It is advisable to design posters on a few key points. Some of the key issues in poster presentations include a comprehensive summary of the poster, better quality of diagrammatic data representation.? Recent study has shown that visually appealing representation of data is more likely to attract an audience than a poster filled with text. ${ }^{8}$ The poster should be balanced in content such as text, data, diagrammatic representation, and adequate empty space to make sure it is not overcrowded. A very important aspect that needs to be noted is the font size that is used. Audiences usually like posters that can be read from at least 3-6 feet.

Basic sciences researchers are generally cautious while presenting their findings. Experiments involving cell culture and animal work take longer time when compared to clinical data. Many authors are cautious in the work they present in view of competition, more so if the data is novel and is in the preli-

Associate Editor of Original Articles International Journal of Medical Students (IJMS). National Institute of Mental Health and Neurosciences, Bangalore, Karnataka, India. ${ }^{2}$ Baylor College of Medicine (NT), Houston, Texas, USA

${ }^{3}$ University of Alabama at Birmingham, USA.

${ }^{4}$ Scientific Editor IJMS. Jordan University of Science and Technology, Ar Ramtha, Irbid, Jordan

${ }^{5}$ Editor in Chief IJMS. Cisalva Institute, School of Public Health, University of Valle, Cali, Colombia.

Correspondence:

Abhishekh H.A. MBBS

Address: Hosur Road, Lakkasandra, Hombegowda Nagar, Bangalore, Karnataka 560029, India

Email: associate.editor.O2@ijms.info 
minary stages.

Whatever the type of presentation is, these are some important points to include: brief introduction, general methodology, results and brief discussion. Points such as Abstract, detailed methods or many references are unnecessary. The Title should attract reader's/listener's interest, summarize key findings and include less than 20 words. Avoid using questions, abbreviations or "new" or "novel" in the title. Readers can refer Wood and Morrison (2011) article for the example poster format. ${ }^{6}$ Presenters are advised to prepare few handouts although it is not absolutely required.

A few studies were conducted to assess the discrepancy in the results presented in conference and subsequently published full length articles. Results showed significant disparity, suggesting the need for precaution while concluding/ making clinical decisions based on findings presented in poster form at a conference. ${ }^{9}$ It cannot be assumed that conference attendees will always understand that the data presented in a poster or oral talk are many times preliminary and incomplete, and do not represent the entire picture. Authors should be aware of this issue and be sure to clarify when findings are due for further testing and validation.

Researchers in developing countries may encounter the additional challenge of getting funding for the travel. It should be noted that most of the international conferences have funding opportunities for minorities and scientists from developing countries. A few of the international organizations, for example International Brain Research Organization, have opportunities to fund the researchers (Available from: http://ibro.info/professional-development/funding-programmes/travel-grants/. Cited 2014 Jun 23).

In conclusion, poster presentations are very important learning opportunities in the career of a budding scientist. Beyond helping in the early dissemination of study data, the presentation of posters offers a chance to make excellent contacts among conference attendees, and greatly improves the curriculum vitae.

\section{References}

1. Deonandan R, Gomes J, Lavigne E, Dinh T, Blanchard R. A pilot study: research poster presentations as an educational tool for undergraduate epidemiology students. Adv Med Educ Pract. 2013 Sep 23;4:183-8.

2. Carmody S, Meier D, Billings JA, Weissman DE, Arnold RM. Training of palliative medicine fellows: a report from the field. J Palliat Med. 2005 Oct;8(5):1005-15.

3. Mileder LP. Medical students and research: Is there a current discrepancy between education and demands? GMS Z Med Ausbild. 2014 May 15;31(2):Doc15.

4. Bonilla-Velez J, Peña-Oscuvilca A, Sahin I, Córdoba-Grueso WS, Fernandez-Zapico ME. The International Journal of Medical Students, a Platform for Medical Student Research Worldwide. Int J Med Students 2013;1(1):6-7.

5. Keshavan V, Tandon N. How to give an effective presentation. Asian J Psychiatr. 2012 Dec;5(4):360-1.

6. Wood CJ, Morrison RS. Writing abstracts and developing posters for national meetings. J Palliat Med. 2011 Mar;14(3):353-9.

7. Miller JE. Preparing and presenting effective research posters. Health Serv Res. 2007 Feb;42(1 Pt 1):311-28.

8. Goodhand JR, Giles CL, Wahed M, Irving PM, Langmead L, Rampton DS. PoSter presentations at medical conferences: an effective way of disseminating research?. Clin Med. 2011 Apr;11(2):138-41.

9. Falagas ME, Rosmarakis ES. Clinical decision-making based on findings presented in conference abstracts: is it safe for our patients? Eur Heart J. 2006 Sep;27(17):2038-9.

\section{Acknowledgments}

None.

Conflict of Interest Statement a Funding

The Authors have no funding, financial relationships or conflicts of interest to disclose.

Cite as:

Abhishekh HA, Tandon N, Rangarajan S, Gharaibeh A, Bonilla-Escobar FJ. Poster Presentations: A Presenting Opportunity in Conferences for Medical Students. Int J Med Students. 2014 Mar-Jun;2(2):38-9. 